\title{
Research Article \\ Study on the Dynamics of Local Pressure Boosting Pneumatic System
}

\author{
Yan Shi, Guanwei Jia, Maolin Cai, and Weiqing Xu \\ School of Automation Science and Electrical Engineering, Beihang University, Beijing 100191, China \\ Correspondence should be addressed to Yan Shi; yesoyou@gmail.com
}

Received 1 October 2014; Revised 25 December 2014; Accepted 11 January 2015

Academic Editor: Stanisław Migorski

Copyright ( 2015 Yan Shi et al. This is an open access article distributed under the Creative Commons Attribution License, which permits unrestricted use, distribution, and reproduction in any medium, provided the original work is properly cited.

Local pressure boosting system is a complex and switched system, which is widely used in modern pneumatic systems, to optimize local pressure boosting system; firstly, the basic and the dimensionless mathematical models of the local pressure system were setup. Furthermore, the mathematical models were verified through the experimental study on the local pressure boosting system. Moreover, the influences of the tank's three main parameters on the performance of local pressure boosting system were studied. It can be seen that the pressure wave amplitude is mainly affected by the dimensionless volume of the tank; its influence degree is $95.1 \%$, and it increases when the later one decreases. The pressure loss of the tank is mainly affected by the dimensionless output pressure, and its influence degree is $68.7 \%$, and it decreases rapidly with the increase of the dimensionless output pressure of the tank. Last, the optimization method of the local pressure boosting system was obtained.

\section{Introduction}

Pressure-boosting technologies have been used in many fields [1-6]. Because of its compact structure, small size, and nonexternal power supply, and so forth, air-driven booster was widely used in locally pressure-boosting occasions of pneumatic system [1-7]. Most common booster was called input pressure reduced pneumatic booster (short for IPR booster), pressure of output air of which was set by adjusting input pressure of the driving-chamber, such as VBA series of booster of SMC Corporation [7]. With the development of energy-saving technologies of pneumatic system, importance of booster has become more and more obvious $[8,9]$. However this type of booster has its own shortages, such as its small output flow, when boosting ratio is higher, the shortage becomes more distinct. Furthermore, its energy efficiency is not high, which restricts its application $[10,11]$.

Recently, main research on pneumatic booster was focused on its characteristics and factors which influence the characteristics of pneumatic booster [12-14]. Moreover, some new structures of pneumatic booster were designed.
However, efficiency and flow of pneumatic booster still were not developed sufficiently [11, 15-17].

Therefore, one novel type of booster was proposed, which is called expansion energy used pneumatic booster, short for EEU booster. The wave amplitude of output flow and pressure increases with the increase of the EEU booster's efficiency and power, and that not only affects the working performance of pneumatic actuator, but also seriously shortens the working span of pneumatic components (such as actuator, regulator, and valve)

To reduce the pulse and the loss of output pressure of the EEU booster, in this paper, the local pressure boosting system (consists of the EEU booster, tank, and pipes, etc.) was studied. Firstly, the working principles of the local pressure boosting system and the EEU booster was introduced, the basic and the dimensionless mathematical models of the local pressure system were set up. Furthermore, pressure boosting pneumatic system was studied experimentally to verify the mathematical models. Last, the influences of the tank's main parameters on the performance of pressure boosting system were studied. This research lays a foundation 


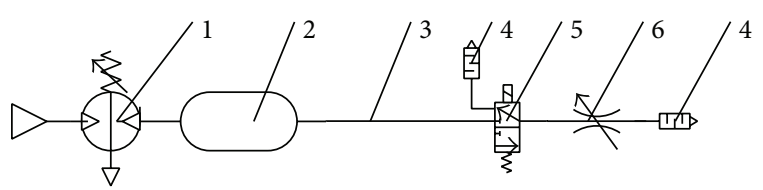

FIgURE 1: Configuration of local pressure boosting system. 1: EEU booster, 2: air tank, 3: pipeline, 4: silencer, 5: solenoid valve, and 6: throttle valve.

for the optimization and energy saving of the local pressure boosting system.

There are several definitions that should be introduced.

(1) Wave Amplitude Ratio. It is the amplitude ratio of the output pressure wave and the input pressure wave of the research unit, which can be used to directly illustrate the damping of pressure pulse of compressed air.

(2) Influence Degree. When a parameter changes in a range, the difference between the maximum value and the minimum value of a characteristic of the research unit is defined as Influence Degree, which represents the degree of the influence of a parameter on a characteristic of the research unit.

(3) Wave Amplitude Influence Ratio. It is the ratio of "the Influence Degree of one parameter on the Wave Amplitude of the research unit" and "the sum of the Influence Degree of other parameters on the Wave Amplitude of the research unit."

(4) Pressure Loss Influence Ratio. It is the ratio of "the Influence Degree of one parameter on the pressure loss of the research unit" and "the sum of the Influence Degree of other parameters on the pressure loss of the research unit."

\section{Working Principle Introduction}

2.1. Working Principles of Pressure Boosting System. The structure of local pressure boosting system can be shown in Figure 1.

As is shown in Figure 1, the research local pressure boosting system is mainly constituted of an EEU booster, a tank, pipes, a solenoid valve, and a throttle valve. The compressed air with low pressure is boosted through the EEU booster and flows into the tank, and pressure pulse of compressed air with high pressure is reduced in the tank, and last, the pressure boosted air is emitted to the atmosphere through the solenoid valve, throttle valve, and the silencer.

2.2. Working Principles of EEU Booster. A typical EEU booster, as shown in Figure 2, is composed of a controller, four magnetic switches, two magnetic rings, two solenoid valves, a piston, two driving chambers, two boosting chambers, and four check valves, and so forth. Input flow of the booster is controlled by the controller.

When piston reaches the left travel destination, Solenoid Valve A and Solenoid Valve B change their position, driving chamber B is connected with the atmosphere, compressed air charged from the primary side flows into boosting chamber A and driving chamber A. Compressed air in boosting chamber $\mathrm{A}$ and driving chamber A drives the piston to move toward the right; then the pressure of air in boosting chamber B increases, and higher-pressure compressed air in boosting chamber B is not discharged from the second side until the pressure is higher than the pressure in the second side.

When the piston moves near the magnetic switch $\mathrm{C}$, the solenoid valve A changes its position and compressed air charged from the primary side stops to flow into driving chamber A, depending on its expansion, compressed air in driving chamber A keeps driving piston to move, and finally, arrives the right travel destination (viz., near the magnetic switch D); then part of expansion energy of the air is used. The solenoid valve A and the solenoid valve B are controlled by the controller to change their position, air in driving chamber A flows to atmosphere, and air, charged from the primary side, flows into boosting chamber $\mathrm{B}$ and driving chamber $\mathrm{B}$; then air in the boosting chamber B and driving chamber $B$ drives the piston to move towards the left, the pressure of air in boosting chamber A ascends, and finally higher-pressure compressed air in boosting chamber $\mathrm{A}$ is discharged from the second side.

When the piston moves near the magnetic switch $\mathrm{B}$, the solenoid valve $\mathrm{A}$ changes its position and compressed air charged from the primary side stops to flow into driving chamber B; then part of expansion energy of the air is used to drive the piston continue to move, and arrives the left travel destination (viz., near the magnetic switch A). The Solenoid Valve A and the Solenoid Valve B change their position, air in driving chamber B is exhausted to atmosphere. The booster goes around and repeats the process discussed above, higherpressure compressed air is discharged continuously.

The piston stroke, when the driving chambers stopped to charge air, is defined to be Piston Stroke-set $(L L)$, as shown in Figure 3.

\section{Modeling of the Local Pressure Boosting System}

3.1. Basic Mathematical Model. To facilitate this research, the following assumptions were made:

(1) The working fluid (air) of the system follows all ideal gas laws.

(2) There is no leakage between the chambers, the area of the piston rod end is too small to be considered, and the effective areas of all intake and exhaust ports are the same.

(3) Supply temperature is equal to atmosphere temperature.

(4) The flow of air moving into and out of the chambers is a stable one-dimensional flow that is equivalent to the flow of air through the nozzle contraction. 


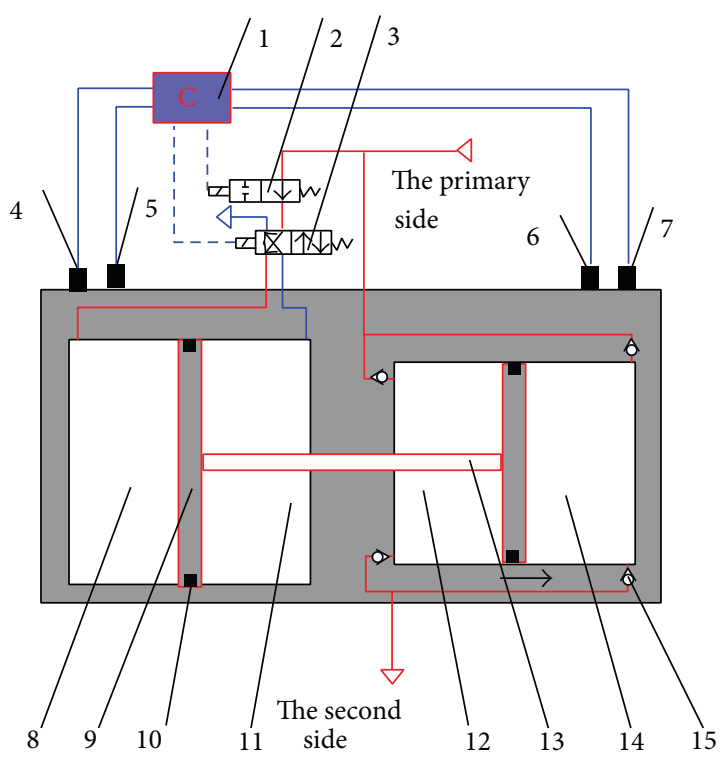

FIGURE 2: Structure of EEU Booster. 1: Controller, 2: solenoid valve $A$, 3: solenoid valve $B$, 4: magnetic switch $A$, 5: magnetic switch $B$, 6: magnetic switch $C$, 7: magnetic switch $D, 8$ : driving chamber A, 9: piston, 10: magnetic ring, 11: driving chamber B, 12: boosting chamber A, 13: piston rod, 14: boosting chamber B, and 15: check valve.

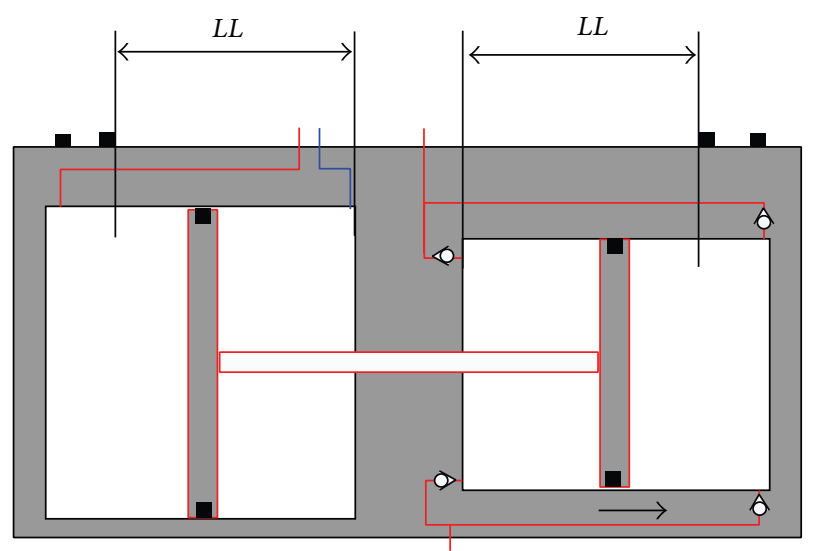

Figure 3: Piston stroke-set of EEU booster.

3.1.1. Energy Equation. Because there is no leakage in either chamber, the chambers do not charge and exhaust air simultaneously. Consequently, the energy equation for the discharge and charge side of each chamber can be illustrated by the following equations:

$$
\begin{gathered}
C_{v} W \frac{d \theta}{d t}=\left(S \cdot h_{c}+C_{v} \cdot G\right)\left(\theta_{a}-\theta\right)+R G \theta_{a}-P A u, \\
C_{v} W \frac{d \theta}{d t}=S \cdot h_{d}\left(\theta_{a}-\theta\right)+R G \theta-P A u .
\end{gathered}
$$

The value of $C_{v}$ is 718 .
3.1.2. Equation of Continuity. From the law of mass conservation, air mass can be given as

$$
\frac{d W}{d t}=G
$$

Air mass flow is calculated from the flow equation, which is described later on.

3.1.3. Flow Equation. The input and output pressures of the booster are below the critical pressure; the temperature of the compressed air is above the critical temperature, according to the ratio $P_{l} / P_{h}$, the flow equation for the flow through a restriction can be written as follows:

$$
G= \begin{cases}\frac{A_{e} P_{h} B}{\sqrt{\theta_{h}}} \varphi\left(P_{h}, P_{l}\right) & \frac{P_{l}}{P_{h}}>b \\ \frac{A_{e} P_{h} D}{\sqrt{\theta_{h}}} & \frac{P_{l}}{P_{h}} \leq b,\end{cases}
$$

where

$$
\begin{gathered}
\varphi\left(P_{h}, P_{l}\right)=\left[\left(\frac{P_{l}}{P_{h}}\right)^{2 / \kappa}-\left(\frac{P_{l}}{P_{h}}\right)^{(\kappa+1) / \kappa}\right], \\
B=\sqrt{\frac{2 \kappa}{R(\kappa-1)}}, \\
D=\left(\frac{2}{\kappa+1}\right)^{1 /(\kappa-1)} \sqrt{\frac{2 \kappa}{R(\kappa+1)}} .
\end{gathered}
$$

The value of $b$, which referenced the critical pressure ratio of the throttle valve, is 0.49 ; the average output flow of the booster is the average value of one period. Consider

$$
\bar{G}=\frac{\int_{0}^{T} G d t}{T} .
$$

3.1.4. Motion Equation. The velocity of the piston is calculated from Newton's second law of motion. In this paper, the friction force model is considered to be the sum of the Coulomb friction and viscous friction. The viscous friction force is considered to be a linear function of piston velocity. The forces on the piston of the booster are shown in Figure 4.

The right side was considered to be the positive direction of the vector. The motion equation of the piston can be given by the following equation:

$$
\frac{d^{2} x}{d t^{2}}= \begin{cases}\frac{1}{M}\left(P_{d A} \cdot A_{d}-P_{d B} \cdot A_{d}+P_{b A} \cdot A_{b}\right. & \\ \left.-P_{b B} \cdot A_{b}-F_{f}\right), & x \neq 0, L \\ 0, & x=0, L,\end{cases}
$$

where

$$
F_{f}= \begin{cases}F_{s} & u=0 \\ F_{c}+C u & u \neq 0 .\end{cases}
$$




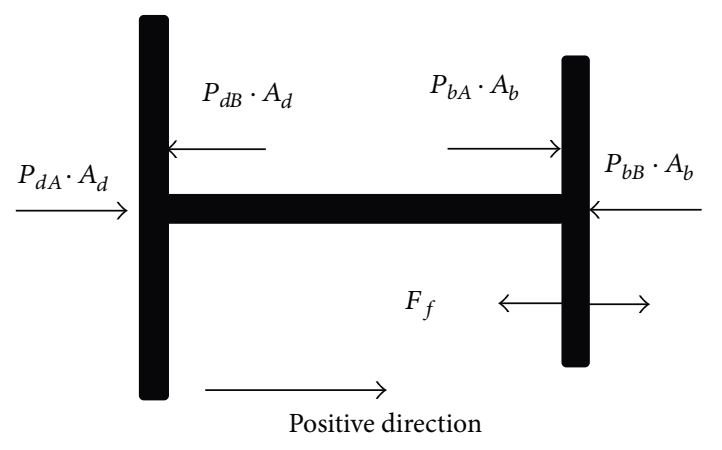

FIgURE 4: The forces on the piston of the booster.

3.1.5. State Equation. Pressure changes in the air in each chamber can be obtained by deriving the state equation of ideal gases:

$$
\frac{d P}{d t}=\frac{1}{V}\left[\frac{P V}{\theta} \cdot \frac{d \theta}{d t}+R \theta G-P A u\right] .
$$

3.2. Dimensionless Mathematical Model. The reference values and the dimensionless variables are shown in Table 1 . The basic mathematical model can be made dimensionless as described in the following section.

3.2.1. Dimensionless Energy Equation. The dimensionless energy equation for the discharge side and the charge side becomes

$$
\begin{aligned}
& W^{*} \frac{d \theta^{*}}{d t^{*}}=\frac{S^{*}}{S_{b}^{*} T_{h d}^{*}}\left(1-\theta^{*}\right)+(k-1)\left(P^{*} u^{*}-G^{*} \theta^{*}\right), \\
& W^{*} \frac{d \theta^{*}}{d t^{*}}=\left(\frac{S^{*}}{S_{b}^{*} T_{h c}^{*}}+G^{*}\right)\left(1-\theta^{*}\right) \\
& +(k-1)\left(G^{*}-P^{*} A^{*} u^{*}\right),
\end{aligned}
$$

respectively, where the dimensionless parameter $T_{h d}^{*}$, which is the dimensionless temperature settling time of the discharge side, is the ratio of the temperature settling time constant, $T_{h d}$, and the isothermal pressure time constant, $T_{p}[18]$. The dimensionless and dimensional time constant can be written as follows:

$$
\begin{gathered}
T_{h d}^{*}=\frac{T_{h d}}{T_{P}}, \\
T_{h d}=\frac{C_{v} W}{S_{b} h_{d}}, \\
S_{b}=2 A_{b}+2 L \sqrt{\pi A_{b}} .
\end{gathered}
$$

The dimensionless maximum heat transfer area can be calculated by the following equation:

$$
S_{b}^{*}=\frac{2 A_{b}+2 L \sqrt{\pi A_{b}}}{A_{b}}=2+2 L \sqrt{\frac{\pi}{A_{b}}} .
$$

For the charge side:

$$
\begin{gathered}
T_{h c}^{*}=\frac{T_{h c}}{T_{P}}, \\
T_{h c}=\frac{C_{v} W}{S_{b} h_{c}} .
\end{gathered}
$$

3.2.2. Dimensionless Equation of Continuity. The dimensionless equation of continuity can be given as the following equation:

$$
\frac{d W^{*}}{d t^{*}}=G^{*}
$$

3.2.3. Dimensionless Flow Equation. The dimensionless flow equation for both sides of the chambers becomes

$$
G^{*}= \begin{cases}\frac{P_{h}^{*} B}{D \sqrt{\theta_{h}^{*}}} \sqrt{\left(\frac{P_{l}^{*}}{P_{h}^{*}}\right)^{2 / \kappa}-\left(\frac{P_{l}^{*}}{P_{h}^{*}}\right)^{(\kappa+1) / \kappa}} & \frac{P_{l}^{*}}{P_{h}^{*}}>0.528 \\ \frac{P_{h}^{*}}{\sqrt{\theta_{h}^{*}}} & \frac{P_{l}^{*}}{P_{h}^{*}} \leq 0.528 .\end{cases}
$$

The average output flow of the booster can be given as

$$
\bar{G}^{*}=\frac{W^{*}}{t^{*}} .
$$

3.2.4. Dimensionless Equation of Motion. The dimensionless equation of motion can be written as follows:

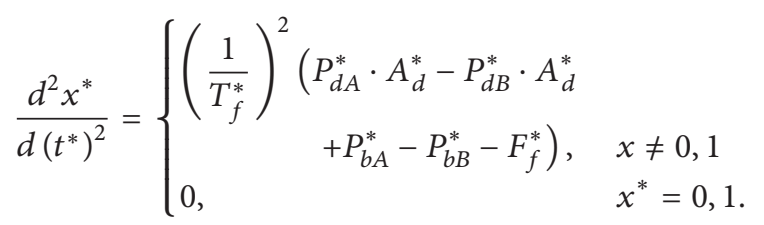

Here, $F_{f}^{*}$ is the dimensionless friction force, which can be written as

$$
F_{f}= \begin{cases}F_{s}^{*} & u^{*}=0 \\ F_{c}^{*}+C^{*} u^{*} & u^{*} \neq 0\end{cases}
$$

Here, $F_{s}^{*}$ is the dimensionless maximum static friction force, $F_{c}^{*}$ is the dimensionless Coulomb friction force, and $C^{*}$ is the dimensional viscous friction force coefficient. All dimensional parameters can be written as follows:

$$
\begin{aligned}
& F_{s}^{*}=\frac{F_{s}}{P_{s} A_{b}}, \\
& F_{c}^{*}=\frac{F_{c}}{P_{s} A_{b}}, \\
& C^{*}=\frac{C \cdot u_{0}}{P_{s} A_{b}} .
\end{aligned}
$$


TABLE 1: Reference values and dimensionless variables.

\begin{tabular}{lccc}
\hline Variable & & Reference value & Dimensionless variable \\
\hline Time & $T_{p}=\frac{W_{b}}{G_{\max }}=\frac{V_{b}}{A_{e} D R \sqrt{\theta_{a}}}$ & Time to totally exhaust $W_{b}$ of air at $G_{\max }$ of air mass flow & $t^{*}=\frac{t}{T_{p}}$ \\
\hline Pressure & $P_{s}$ & Supply pressure & $P^{*}=\frac{P}{P_{s}}$ \\
\hline Temperature & $\theta_{a}$ & Atmosphere temperature & $\theta^{*}=\frac{\theta}{\theta_{a}}$ \\
\hline Air mass flow & $G_{\max }=\frac{A_{e} P_{s} D}{\sqrt{\theta_{s}}}$ & Maximum air mass flow at charge side of boosting chamber & $G^{*}=\frac{G}{G_{\text {max }}}$ \\
\hline Air mass & $W_{b}=\frac{P_{s} V_{b}}{R \theta_{a}}$ & Maximum air mass in boosting chamber & $W^{*}=\frac{W}{W_{b}}=\frac{P^{*} V^{*}}{\theta^{*}}$ \\
\hline Displacement & $L$ & Maximum displacement & $x^{*}=\frac{x}{L}$ \\
\hline Volume & $V_{b}=L \cdot A_{b}$ & Maximum volume of boosting chamber B & $V^{*}=\frac{V}{V_{b}}$ \\
\hline Area of piston & $A_{b}$ & Area of piston in boosting chamber \\
\hline Diameter & $d_{b}$ & Diameter of piston in boosting chamber & $A^{*}=\frac{A}{A_{b}}$ \\
\hline
\end{tabular}

Dimensionless parameter, $T_{f}^{*}$, is defined in the following. $T_{f}^{*}$ corresponds to the $J$-parameter that is used in the current selection method of a pneumatic cylinder $[19,20]$. The $J$ parameter is given by

$$
\begin{gathered}
T_{f}^{*}=\frac{T_{f}}{T_{p}}, \\
T_{f}=\sqrt{\frac{M L}{A_{b} P_{s}},} \\
J=\frac{T_{p}^{2} P_{s} A_{d}}{L M} .
\end{gathered}
$$

From (19), (20), and (21), the relation between $T_{f}^{*}$ and the $J$-parameter is

$$
J=\left(\frac{1}{T_{f}^{*}}\right)^{2} .
$$

Because the $J$-parameter appears as a coefficient of acceleration, it was thought that this parameter related to the inertia of the booster, and it is known as the inertia coefficient. The dimensionless parameter, $T_{f}^{*}$, represents the dimensionless natural period of the booster [21].

The dimensionless Piston Stroke-set $\left(L L^{*}\right)$ is

$$
L L^{*}=\frac{L L}{L} .
$$

3.2.5. Dimensionless State Equation. The dimensionless state equation for the discharge side and charge side becomes

$$
\frac{d P^{*}}{d t^{*}}=\frac{P^{*}}{\theta^{*}} \frac{d \theta^{*}}{d t^{*}}+\frac{\theta^{*} G^{*}}{V^{*}}-\frac{P^{*} A^{*} u^{*}}{V^{*}} .
$$

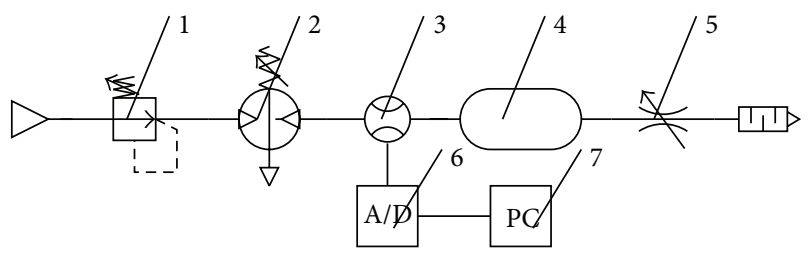

FIGURE 5: Configuration of experimental apparatus. 1: Regulator, 2: booster, 3: air power meter, 4: tank, 5: throttle valve, 6: data acquisition card, and 7: computer.

\section{Experimental Study on the Pressure Boosting System}

The dimensionless output flow and dimensionless cycle time can be attained easily and expediently. These parameters were studied experimentally to verify the dimensionless mathematical model that was set up above.

The experimental apparatus shown in Figure 5 consists of a regulator (IR3020-03BC) by SMC, an EEU booster, PLC (S7-220) by Siemens, a flow sensor (APM-450) by Tokyo Meter, a tank, a throttle valve (AS3001F) by SMC, and a data acquisition card (USB-4711A) by Advantech. The EEU booster is composed of two cylinders (CDQ2B100-100D), a floating connector (JAL100-26-150), four magnetic switches (A73), and four check valves (AKH08) by SMC. The adopted flow sensor is an Air Power Meter (integrates a thermal type flow sensor, a semiconductor type pressure sensor, and a thermocouple type temperature sensor), which can measure the pressure, flow, and temperature of compressed air simultaneously. The accuracy of pressure, flow, and temperature is $0.1 \%, \pm 1 \%$ F.S. and $0.10 \mathrm{C}$, respectively $[22,23]$. 
TABLE 2: The initial values of the parameters.

\begin{tabular}{llcccccccc}
\hline Parameter & $L L^{*}$ & $P_{o}^{*}$ & $A^{*}$ & $F_{f}^{*}$ & $S_{b}^{*}$ & $C^{*}$ & $T_{f}^{*}$ & $T_{h c}^{*}$ & $T_{h d}^{*}$ \\
\hline Value & 0.67 & 1.33 & 1 & 0.008 & 1 & 0.0008 & 0.0167 & 4.47 & 2.98 \\
\hline
\end{tabular}

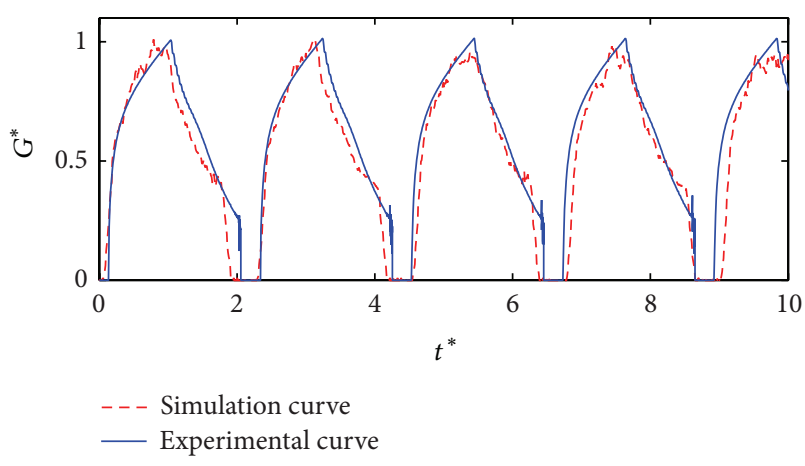

Figure 6: Curves of output flow of boosters.

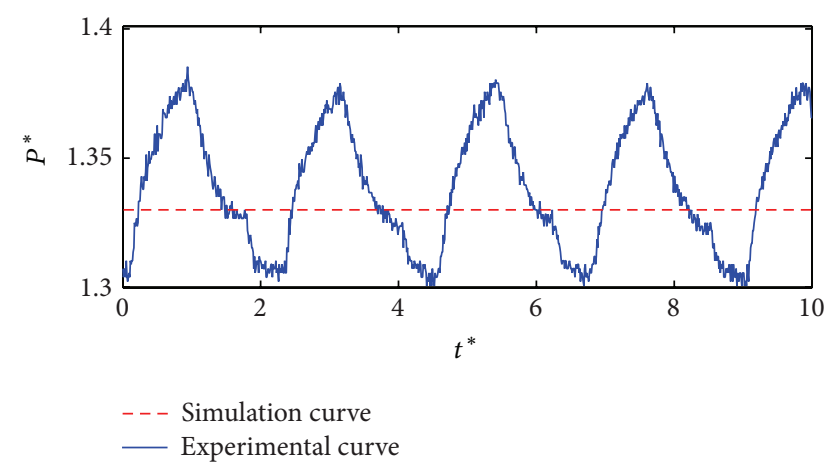

Figure 7: Curves of pressure of air in tank.

In this experiment, we first opened the compressed air source, adjusted the regulator, and set the pressure to the fixed value (0.6 MPa). Next, we adjusted the throttle valve, making sure that air was exhausted from the tank steadily, and the pressure of the air was approximately the fixed value $(0.8 \mathrm{MPa})$. The last stage was data acquisition and preservation.

The values of the nine dimensionless parameters are shown in Table 2. The experiment can be performed according to the method described above. The output flow of the booster, which was obtained by simulation and experimentation, is shown in Figure 6, and the pressure curve of the compressed air in the tank is shown in Figure 7. As the dimensionless output pressure was set to a fixed value (1.33), therefore the simulation curve of pressure of air in tank is a line.

From Figures 9 and 10, it is clear that the simulation results are more consistent with the experimental results, and this verifies the mathematical model above. It should be noted that the effective area of intake and exhaust port is difficult to be determined, so the parameters were adjusted to get the good fit.
The main reasons for the difference between the simulation results and the experimental results are the fluctuation of the supply pressure, leakage between chambers, temperature of the atmosphere, and the fluctuation of pressure of air in tank, which can be seen in Figure 7.

\section{Study on the Performance of the Pressure Boosting System}

5.1. The Influence of the Dimensionless Volume of the Tank. Assume that the dimensionless output pressure of the tank is 1.33, the dimensionless piston stroke-set of the tank is 0.83 , the dimensionless diameter of the outlet of the tank is 1 , and the dimensionless volume of the tank is set 1.4, 9.9, 14.2, $21.2,28.3,35.4$, and 42.4 , respectively. The research results are shown in Figure 8.

As shown in Figure 8, the tank has a good inhibiting effect on the output pressure pulse; pressure wave amplitude in the tank increases with the decrease of the dimensionless volume of the tank, and the pressure loss of the tank increase with the decease of the volume of the tank.

5.2. The Influence of the Dimensionless Output Pressure of the Tank. Assume that the dimensionless volume of the tank is 11.33, the dimensionless piston stroke-set of the tank is 0.83 , the dimensionless diameter of the outlet of the tank is 1 , and the dimensionless output pressure of the tank is set 1.3, 1.29, $1.33,1.38,1.42,1.46$, and 1.5 , respectively. The research results are shown in Figure 9.

As shown in Figure 9, the pressure wave amplitude in the tank increases slowly with the increase of the dimensionless output pressure of the tank, and the pressure loss of the tank decreases rapidly with the increase of the output pressure of the tank.

5.3. The Influence of the Dimensionless Diameter of the Outlet of the Tank. Assume that the dimensionless volume of the tank is 11.33 , the dimensionless piston stroke-set of the tank is 0.83 , the dimensionless output pressure of the tank is 1.33 , and the dimensionless diameter of the outlet of the tank is set $0.8,0.9,1,1.1,1.2$, and 1.3 , respectively, the research results are shown in Figure 10.

As shown in Figure 10, the pressure wave amplitude in the tank rarely has respond to the changes of the dimensionless diameter of the outlet of the tank, and the pressure loss of the tank increase with the increase of the dimensionless diameter of the outlet of the tank.

5.4. The Compare of the Influence of the Three Main Parameters of the Tank. Each parameter can be changed within a certain range for comparison of the influence degree while other 


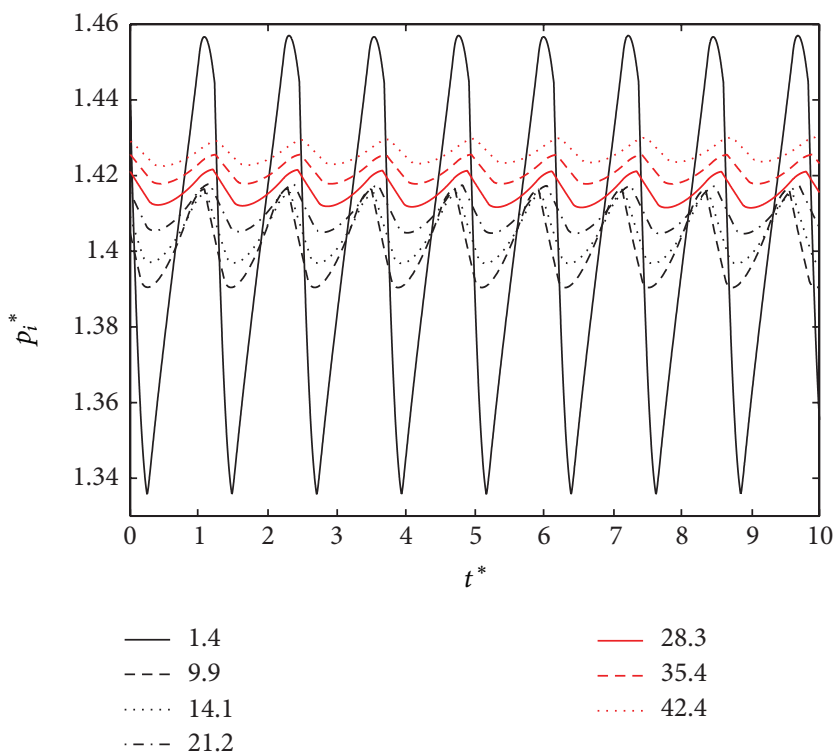

(a) The dimensionless dynamic characteristics of air pressure in the tank

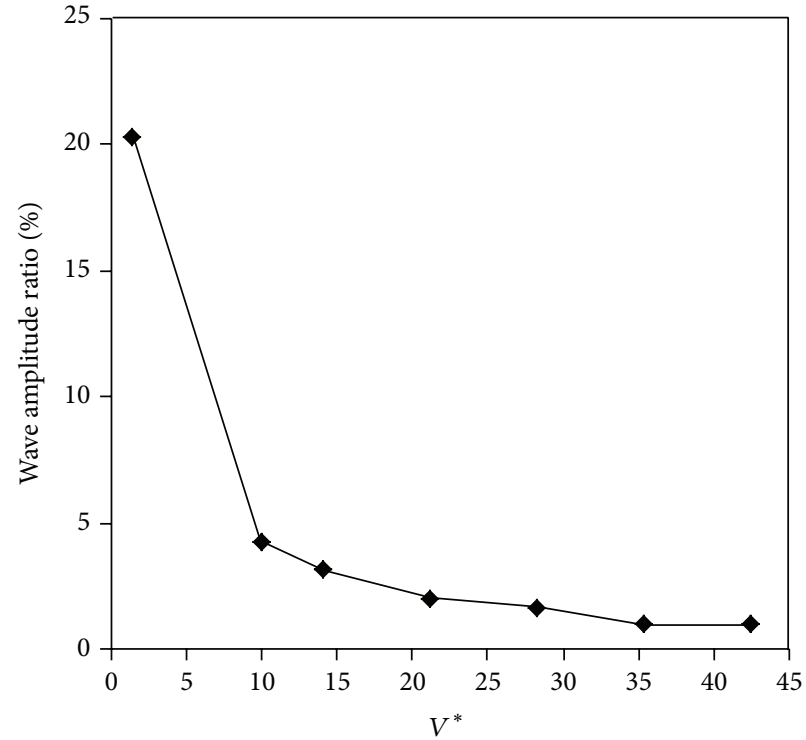

(b) The dimensionless pressure wave amplitude ratio of the tank

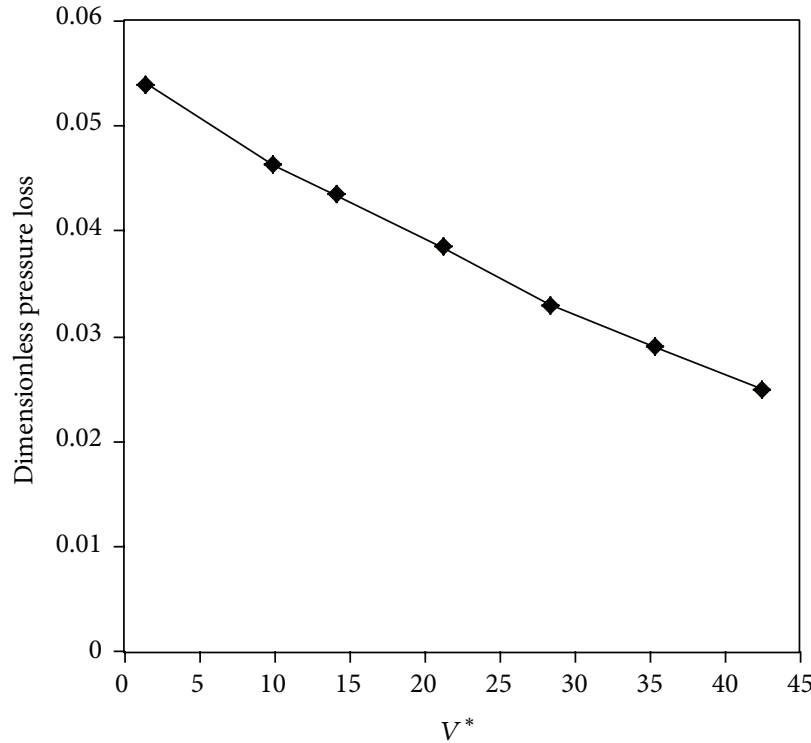

(c) The dimensionless pressure loss in the tank

FIGURE 8: The influence of the dimensionless volume of the tank.

parameters are kept constant. And the influence degree of the main parameters of the tank on the pressure wave amplitude and the pressure loss influence ratio can be given by the following equations:

$$
\begin{gathered}
p_{\text {wa-total }}=p_{\text {wa- } V^{*}}+p_{\text {wa- } p_{o}^{*}}+p_{\text {wa- }-d_{o}^{*}}, \\
\operatorname{Dg}_{\text {wa- } V^{*}}=\frac{p_{\text {wa- }-V^{*}}}{p_{\text {wa-total }}} \\
\operatorname{Dg}_{\text {wa- } V^{*}}=\frac{p_{\text {wa- }-p_{o}^{*}}}{p_{\text {wa-total }}}
\end{gathered}
$$




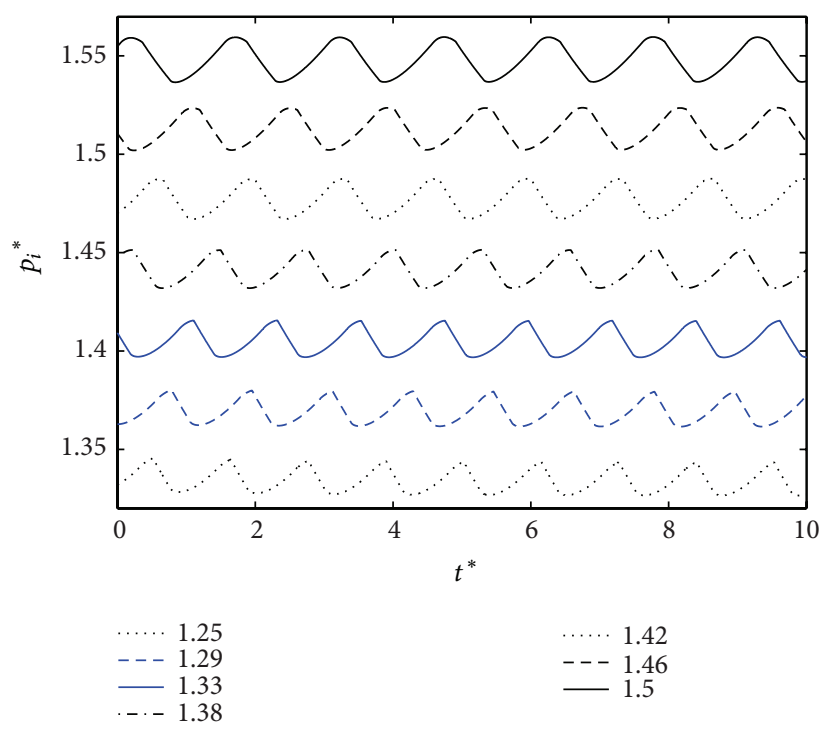

(a) The dimensionless dynamic characteristics of pressure in the tank

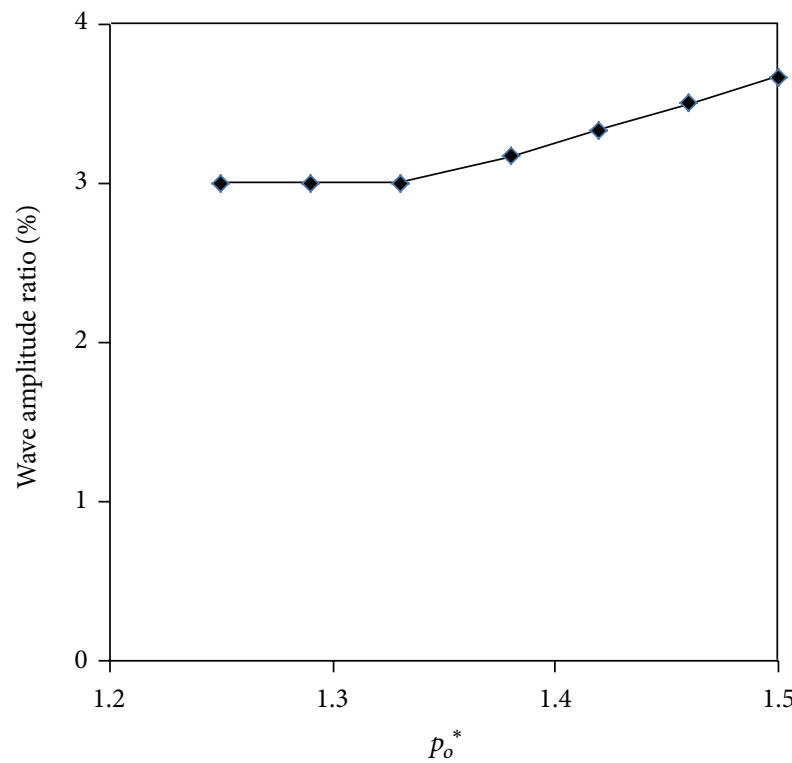

(b) The dimensionless pressure wave amplitude in the tank

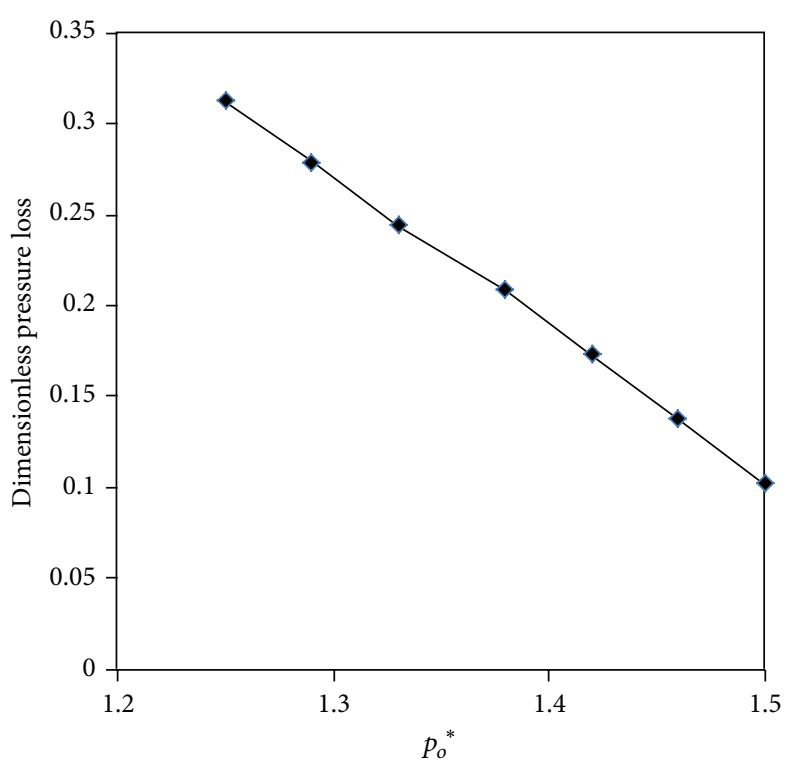

(c) The dimensionless pressure loss in the tank

FIGURE 9: The influence of the dimensionless output pressure of the tank.

Based on the numerical study, the influence degrees of the dimensionless volume, the dimensionless output pressure, and the dimensionless diameter of the outlet of the tank on the pressure wave amplitude and the pressure loss are shown in Figures 11 and 12.

As shown in Figures 11 and 12, it is clear that

(1) The pressure wave amplitude of the tank is mainly affected by the dimensionless volume of the tank, and its influence degree is $95.1 \%$. And the influences of the dimensionless output pressure and the diameter of the outlet of the tank can be ignored.
(2) The pressure loss of the tank is mainly affected by the dimensionless output pressure and the diameter of the outlet of the tank, and the three parameters' influence degree is $68.7 \%, 21.8 \%$, and $9.5 \%$.

\section{Conclusions}

In this paper, a new kind of booster, EEU booster, was proposed, the local pressure boosting system was studied, and the basic mathematical model of the system was developed. Appropriate reference values were selected, the basic mathematical model was transferred to a dimensionless expression, 


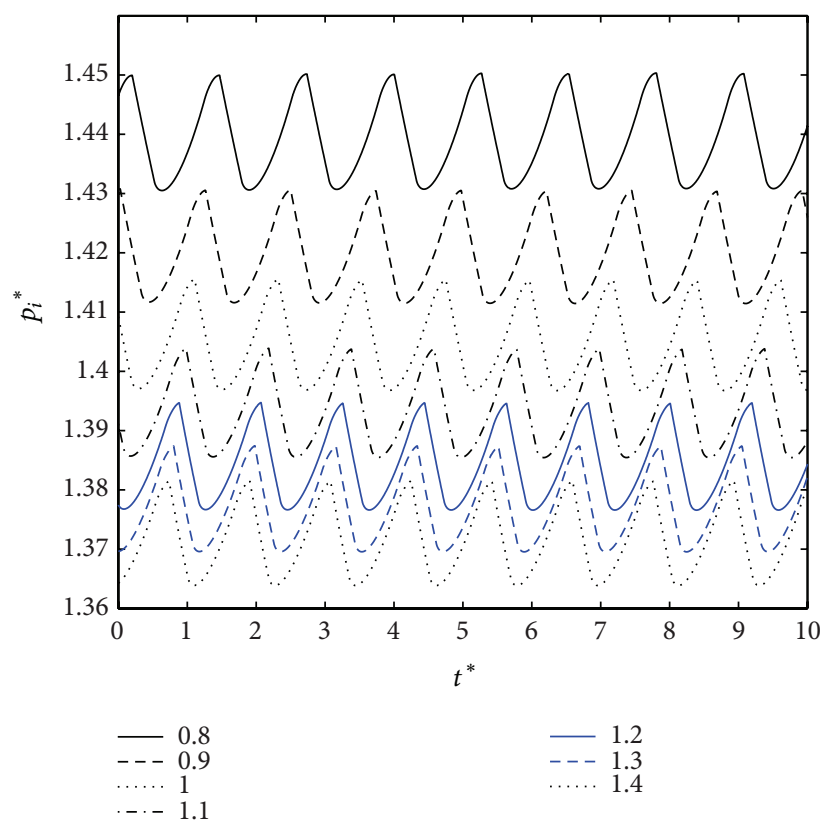

(a) The dimensionless dynamic characteristics of pressure in the tank

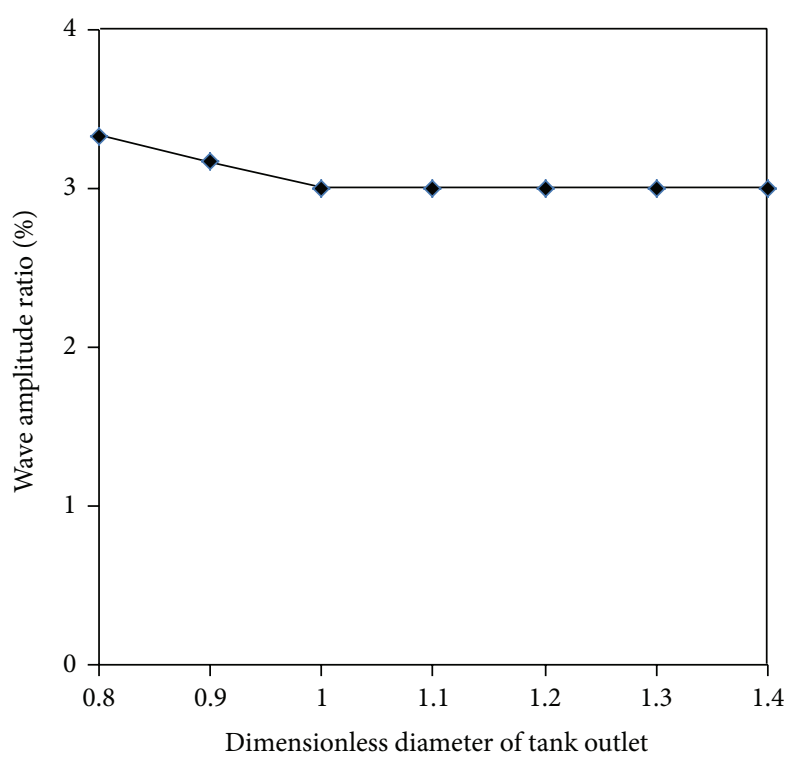

(b) The dimensionless pressure wave amplitude in the tank

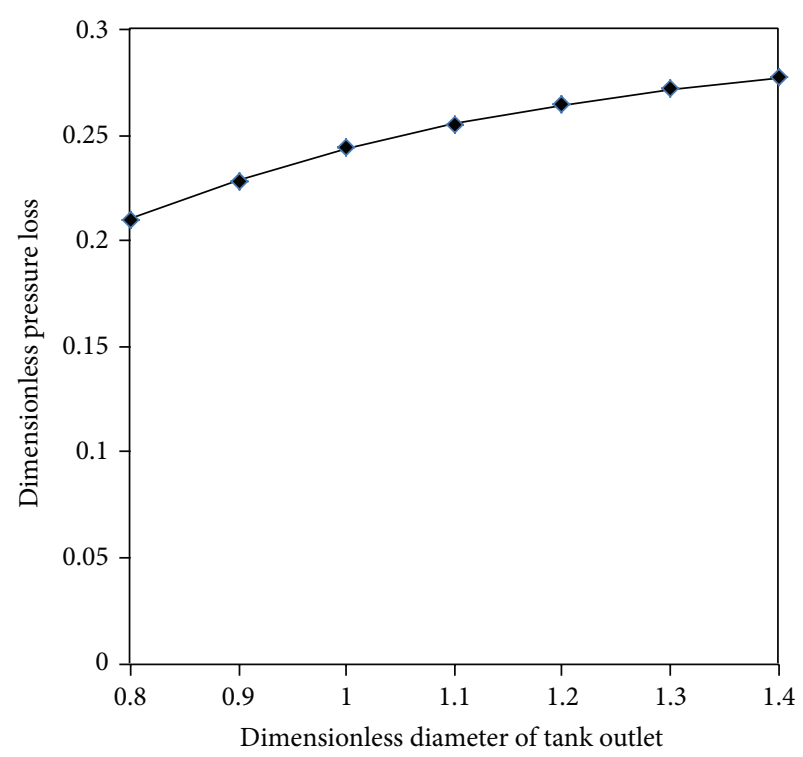

(c) The dimensionless pressure loss in the tank

FIGURE 10: The influence of the dimensionless diameter of the outlet of the tank.

and the influence of the tank on the working performance of the local pressure boosting system was analyzed through simulation and experimentation. The conclusions are summarized as follows.

(1) Simulation results have better consistency with experimental results, and the mathematical model set up above is correct.

(2) The pressure wave amplitude is mainly affected by the dimensionless volume of the tank, its influence degree is $95.1 \%$, and it increases when the later one decreases. Other parameters' influence on pressure wave amplitude can be ignored.
(3) The pressure loss of the tank is mainly affected by the dimensionless output pressure, and secondly by the diameter of the outlet of the tank, and the three parameters' influence degree is $68.7 \%, 21.8 \%$, and $9.5 \%$.

(4) The pressure loss of the compressed air decreases with the increase of the volume of the tank and rapidly decreases with the increase of the dimensionless output pressure of the tank and increases with the increase of the dimensionless diameter of the outlet of the tank.

So, aiming at the optimization of the local pressure boosting pneumatic system, we can draw conclusions as follows. 


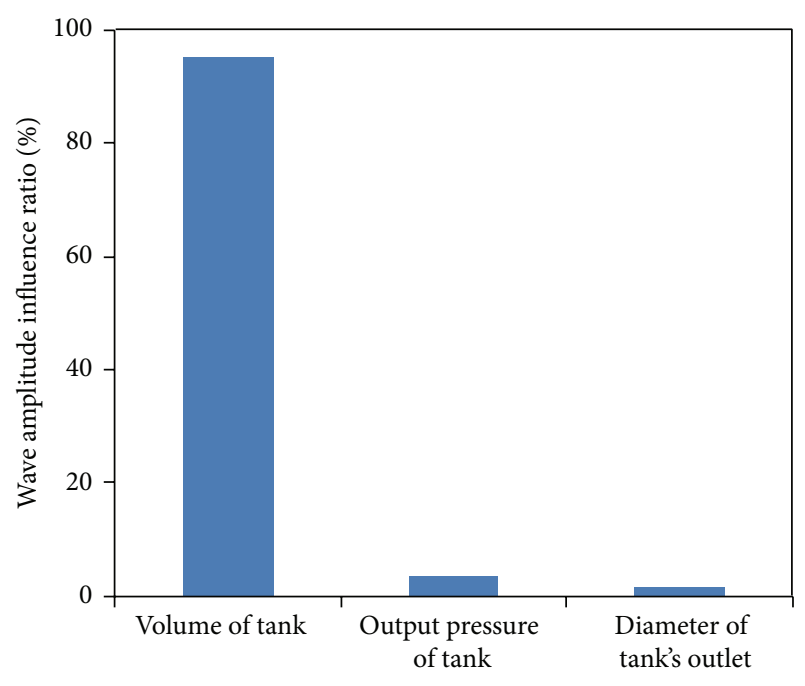

FIGURE 11: The influence degree of the main parameters of the tank on the pressure wave amplitude.

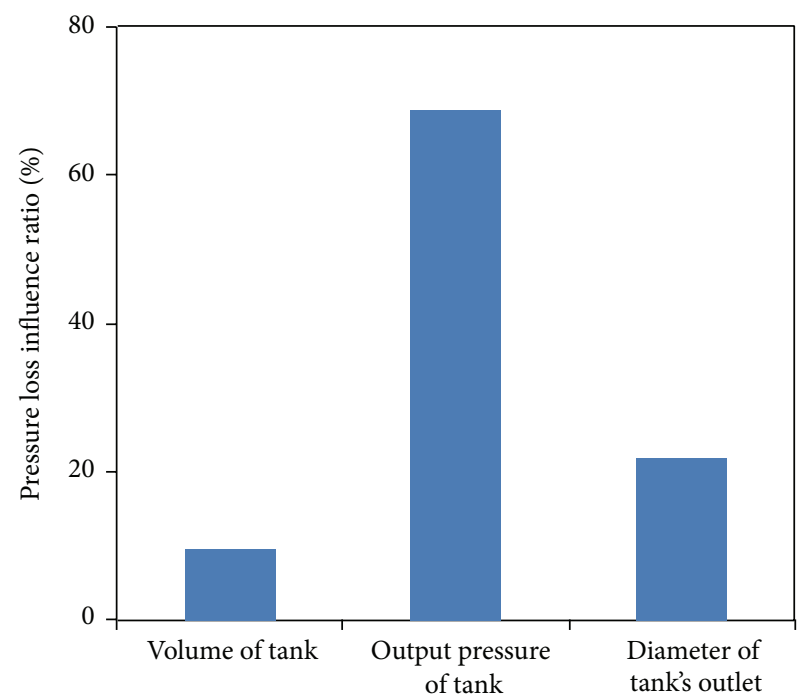

FIGURE 12: The influence degree of the main parameters of the tank on the pressure loss.

(1) In the case of allowing, increase the volume of the tank as much as possible.

(2) In the case that the industrial site requirements have been satisfied, increase the output pressure in a proper range.

(3) In the case that the booster is working in its best condition, decrease the diameter of the outlet of the tank and decrease the output flow rate of the tank in a proper range.

If those measurements were taken, the pressure wave amplitude in the tank and the pressure loss of the compressed air could both be reduced.

This research lays a foundation for the optimization design and energy saving of the local pressure boosting system.

\section{Nomenclature}

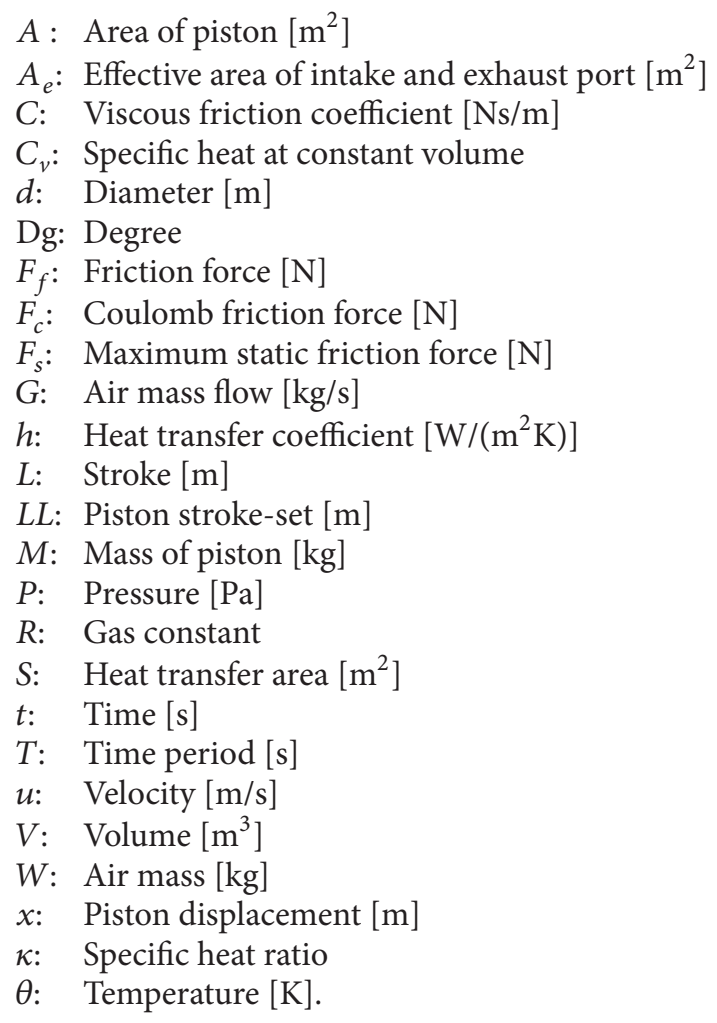

\section{Subscripts}

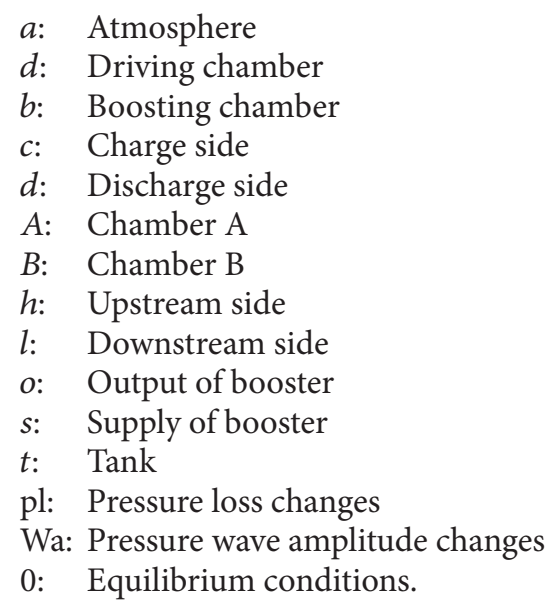

\section{Superscripts}

*: Dimensionless.

\section{Conflict of Interests}

The authors declare that there is no conflict of interests regarding the publication of this paper. 


\section{Acknowledgment}

This project is supported by National Natural Science Foundation of China Grant no. (51205008).

\section{References}

[1] Z. Li, Y. Zhao, L. Li, and P. Shu, "Mathematical modeling of compression processes in air-driven boosters," Applied Thermal Engineering, vol. 27, no. 8-9, pp. 1516-1521, 2007.

[2] H. P. Cheng, C. J. Chen, and P. W. Cheng, "Computational fluid dynamics performance estimation of turbo booster vacuum pump," Journal of Fluids Engineering, vol. 125, no. 3, pp. 586589, 2003.

[3] C. A. M. Brenninkmeijer and P. A. Roberts, "An air-driven pressure booster pump for aircraft-based air sampling," Journal of Atmospheric and Oceanic Technology, vol. 11, no. 6, pp. 16641671, 1994.

[4] V. E. Minaichev and V. M. Zykov, "Cryo-codensation booster pump," Instruments and Experimental Techniques, vol. 17, no. 3, pp. 795-798, 1974.

[5] P. Wang, F. X. Ren, and Y. P. Wang, "Balance characteristic of hydraulic balanced booster plunger pump," China Petroleum Machinery, vol. 25, no. 1, pp. 26-28, 1997 (Chinese).

[6] L. J. Budgen, "Developments in the transmission for mechanical booster pumps," Journal of Vacuum Science and Technology A: Vacuum, Surfaces and Films, vol. 1, no. 2, pp. 147-149, 1982.

[7] SMC (China) Co, Modern Practical Pneumatic Technology, China Machine Press, Beijing, China, 2008, (Chinese).

[8] N. Oneyama, "Pneumatic manufacturer's attempts on energy saving," Hydraulics and Pneumatics, vol. 2, no. 3, pp. 372-377, 1996.

[9] Y. Shi, M. Cai, and G. Wang, "Study on air-supplied with different pressure and locally pressure-boosting technology of pneumatic system," Machine Toll and Hydraulics, vol. 28, no. 9, pp. 57-59, 2010.

[10] N. Hamaura, T. Fujita, and T. Kagawa, "Characteristics analysis of pneumatic booster," in Proceedings of the Autumn Symposium on Hydraulics and Pneumatics, pp. 77-80, 1994, (Japanese).

[11] O. Takeuchi, T. Fujita, and T. Kagawa, "Characteristics analysis of expanding-type booster," in Proceedings of the Autumn Symposium on Hydraulics and Pneumatics, pp. 69-72, 1995, (Japanese).

[12] W. Haitao, W. Xiong, and L. Zhonghua, "Research on the dynamic characteristics during boosting charge of gas booster," in Proceedings of the 5th National Conference on Fluid Power Transmission and Control \& Conference on Hydraulic and Pneumatic of Chinese Society of Aeronautics and Astronautics, pp. 362-366, Beijing, China, October 2008, (Chinese).

[13] Xu. Wang, H. Wang, and W. Xiong, "Study on the characteristics of air driven gas booster based on experiments," in Proceedings of the 7th International Conference on Fluid Power Transmission and Control, pp. 608-611, Hangzhou, China, 2009.

[14] S. H. I. Yan and C. A. I. Maolin, "Experimental study on working characteristics of pneumatic booster valve," in Proceedings of the 7th International Conference on Fluid Power Transmission and Control, pp. 511-515, Hangzhou, China, 2009.

[15] F. Dong, G. He, Z. Zhang et al., "Design of pneumatic booster pump," Mechanical Science and Technology, vol. 27, no. 1, pp. 2327, 2008 (Chinese).
[16] X. Wang, Study on New Kind Energy-Saving Gas Booster, Dalian Maritime University, Dalian, China, 2009, (Chinese).

[17] S. H. I. Yan and C. A. I. Maolin, "Study on efficiency and flow characteristics of two kinds of pneumatic booster valves," in Proceedings of the International Conference on Computer, Mechatronics, Control and Electronic Engineering, pp. 45-50, Changchun, China, 2010.

[18] T. Kagawa, "Heat transfer effects on the frequency response of a pneumatic nozzle flapper," Journal of Dynamic Systems, Measurement and Control, vol. 107, no. 4, pp. 332-336, 1985.

[19] K. Ando, Study on Characteristics of Pneumatic Systems for Resistance Welding Machines, The Japan Welding Engineering Society, 1965, (Japanese).

[20] U. Kadota, "Characteristics of air (13)," Hydraulics and Pneumatics Design, vol. 9, no. 2, pp. 109-115, 1971.

[21] K. Tokashiki, Dynamic Characteristics of Pneumatic Cylinder Systems, Department of Control Engineering, Tokyo Institute of Technology, Tokyo, Japan, 1999.

[22] M. Cai, K. Kawashima, and T. Kagawa, "Power assessment of flowing compressed air," Journal of Fluids Engineering, vol. 128, no. 2, pp. 402-405, 2006.

[23] C. Maolin, F. Tatsuya, K. Kenji, and K. Toshiharu, "Development of pneumatic power meter for energy saving," in Proceedings of Symposium on Fluid Power System at Spring, pp. 119-121, Tokyo, Japan, 2003. 


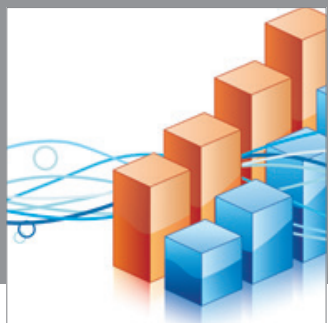

Advances in

Operations Research

mansans

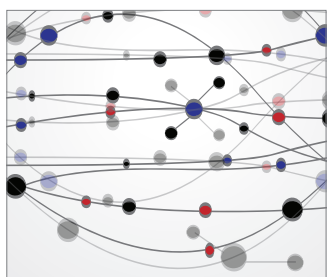

The Scientific World Journal
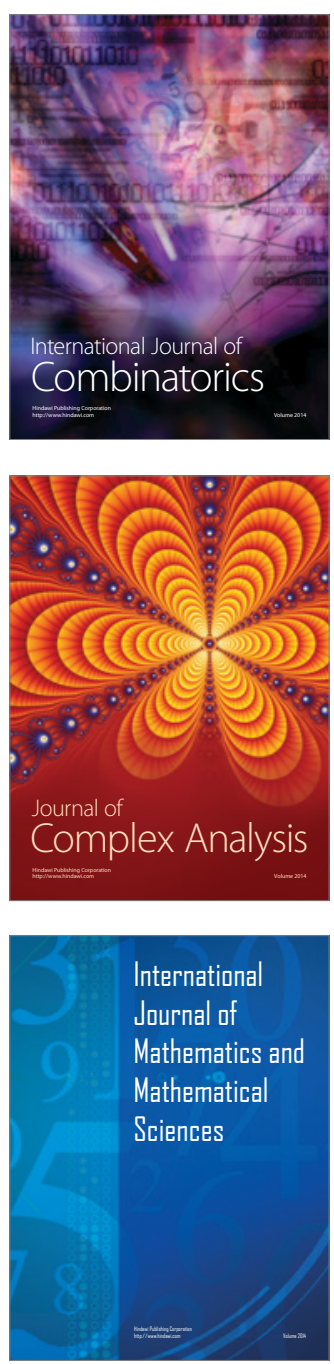
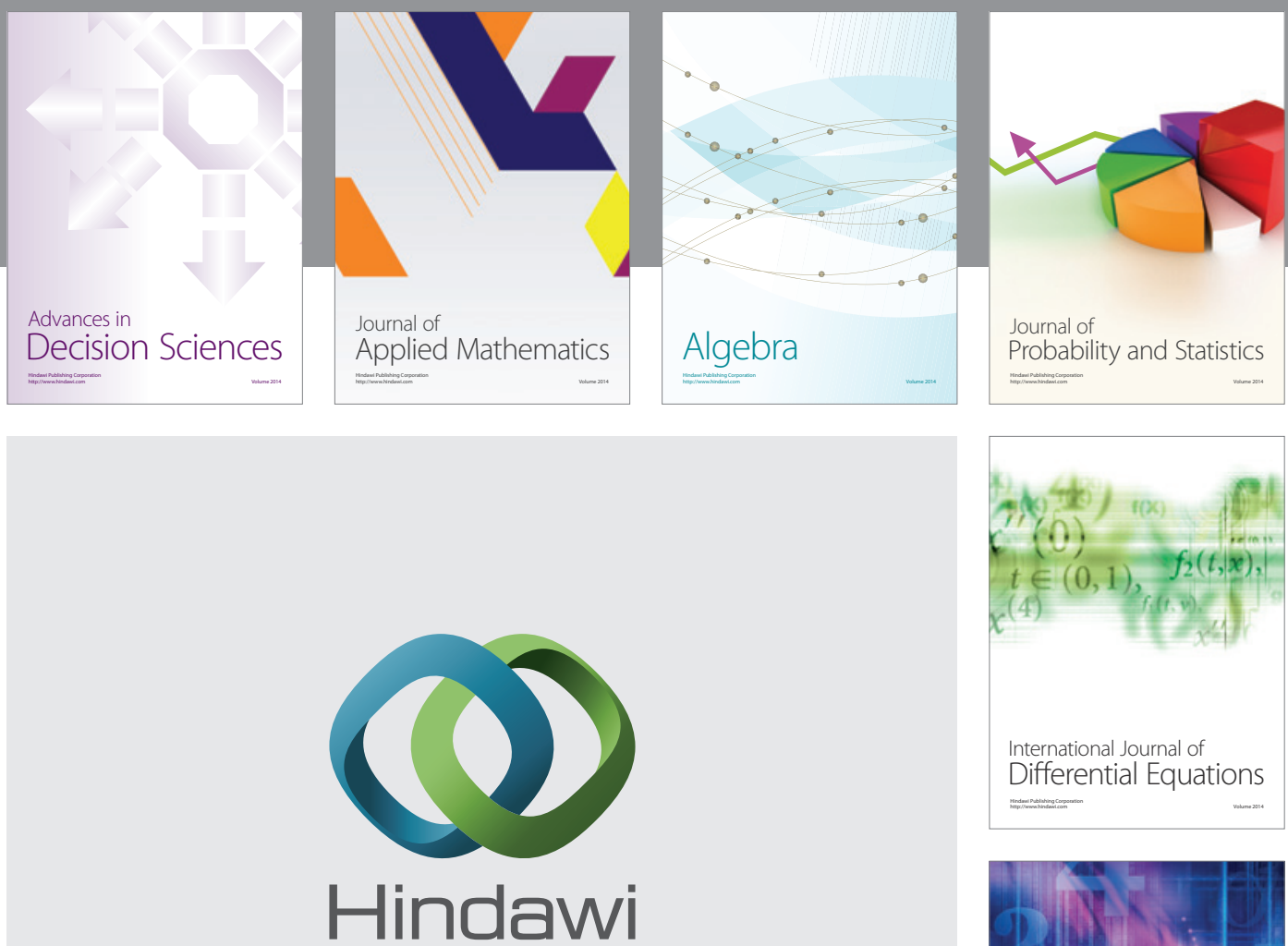

Submit your manuscripts at http://www.hindawi.com
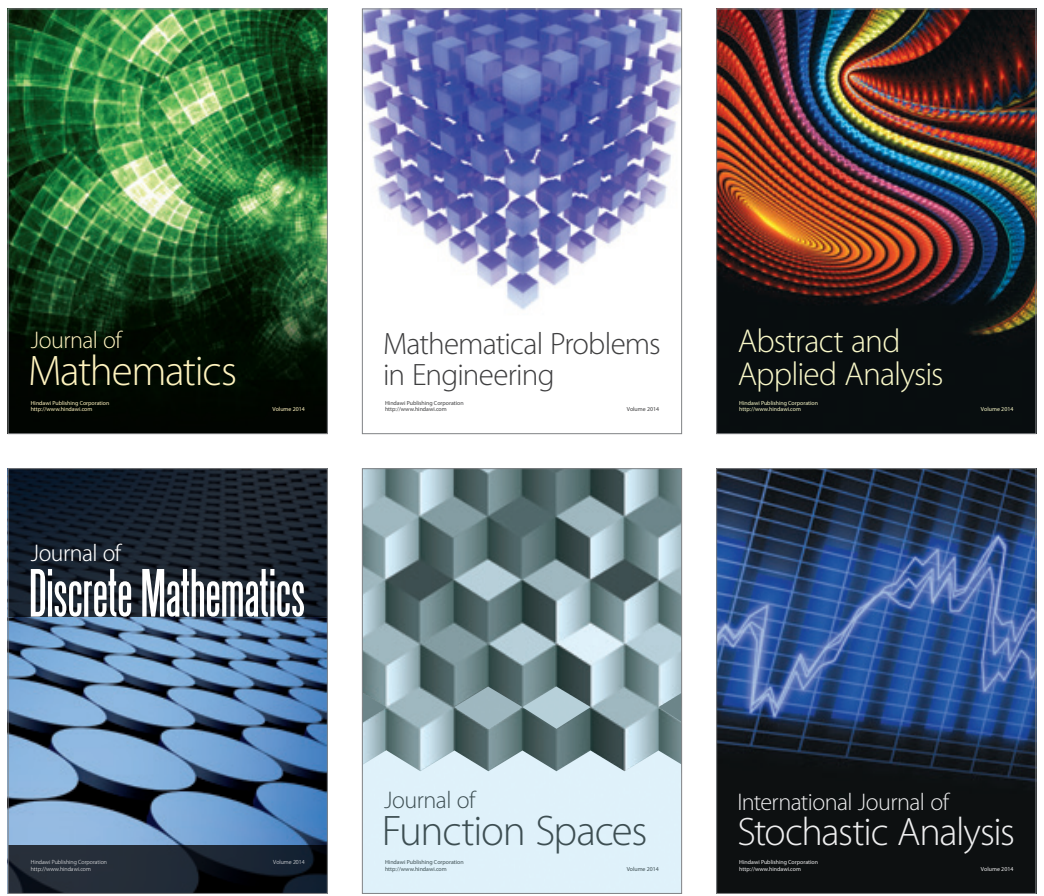

Journal of

Function Spaces

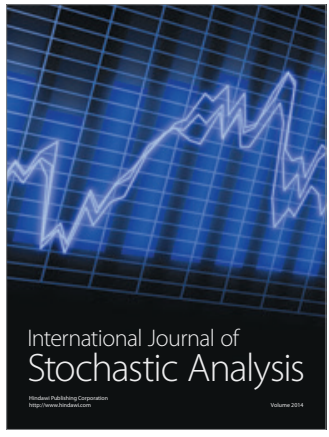

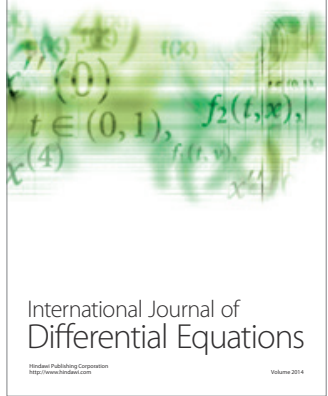
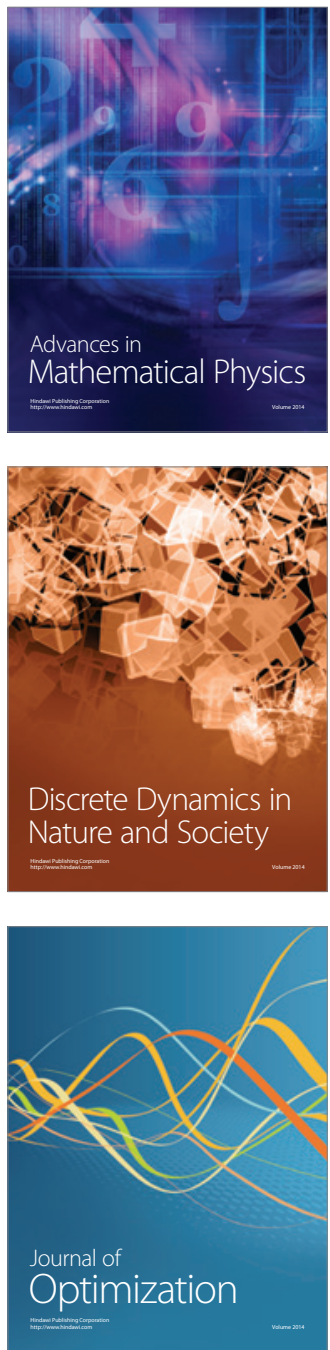